\title{
Prevalence of cardiac morphological and functional alterations in systemic lupus erythematosus patients with a low disease activity
}

\author{
Florian Andre ${ }^{1 *}$, Maria Fernanda Braggion Santos ${ }^{2}$, Hassan Abdel-Aty ${ }^{1}$ \\ From 16th Annual SCMR Scientific Sessions \\ San Francisco, CA, USA. 31 January - 3 February 2013
}

\section{Background}

Cardiac involvement in systemic lupus erythematosus (SLE) is a common complication and is associated with a considerable morbidity and mortality in these patients. As affected individuals often present with subclinical or nonspecific symptoms the betimes confirmation of cardiac manifestations is challenging. Cardiovascular magnetic resonance (CMR) with late gadolinium enhancement (LGE) is the current gold-standard for non-invasive tissue characterization as well as for the evaluation of systolic and diastolic function. In this study we assessed the cardiac morphology and function in SLE patients with low disease activity.

\section{Methods}

We studied twenty-nine SLE patients (3 male, 26 female) fulfilling the SLE diagnostic criteria of the American College of Rheumatology and compared them to thirty agematched healthy volunteers. All patients were in a stable clinical condition and only patients with a low disease activity (SLEDAI Index $<5$ ) were included. All of them received an individually optimized medication. CMR images were acquired on a $1.5 \mathrm{~T}$ whole-body MRI (Achieva, Philips Healthcare, Best, The Netherlands) using a 32-element cardiac phased-array coil. Short and long axis views were obtained applying a standard clinical steady-state free-precession sequence and LGE CMR imaging was performed (Gadolinium-DTPA $0.2 \mathrm{mmol} / \mathrm{kg}$ body weight, Magnevist, Schering, Berlin, Germany) in 27 patients. Left and right ventricular (LV, RV) end-diastolic and end-systolic volumes (EDV, ESV), stroke volume (SV), ejection fraction (EF) as well as the mitral/tricuspid annular plane systolic excursion (M/TAPSE) and LV wall mass were measured.

\section{Results}

SLE patients and healthy controls had comparable demographic and clinical characteristics. SLE patients had significantly higher LV-EDV, LV-ESV and LV-EF. LV-SV and MAPSE did not differ significantly between patients and healthy controls. However SLE patients had significantly higher RV-EDV, RV-ESV, RV-SV, RV-EF and TAPSE values. LV mass showed no significant differences. Nineteen of twenty-seven assessed patients (70\%) showed LGE CMR as a sign of myocardial involvement.

\section{Conclusions}

Even in SLE patients with a low disease activity there is a high prevalence of myocardial and functional alterations. Especially the RV function is affected which could be due to early onset pulmonary manifestations of SLE. Regarding the high morbidity and mortality of cardiac complications in SLE patients, CMR may be an important non-invasive method for the early diagnosis of cardiopulmonary involvement.

\section{Funding}

none. 
Table 1 Comparison of SLE patients to healthy controls

\begin{tabular}{|c|c|c|c|}
\hline & $\begin{array}{l}\text { SLE patients } \\
\quad(\mathrm{n}=29)\end{array}$ & $\begin{array}{l}\text { Healthy controls } \\
\qquad(n=30)\end{array}$ & \\
\hline LV EF (\%) & $64.1 \pm 9.4$ & $68.4 \pm 5.4$ & $p<0.05$ \\
\hline LV EDV (ml) & $152.7 \pm 31.0$ & $129.1 \pm 30.9$ & $p<0.01$ \\
\hline LV ESV (ml) & $56.3 \pm 24.3$ & $41.7 \pm 16.0$ & $p<0.01$ \\
\hline LV SV (ml) & $96.5 \pm 19.5$ & $87.3 \pm 16.8$ & $p=n . s$. \\
\hline $\begin{array}{l}\text { LV Cardiac output (I/ } \\
\text { min) }\end{array}$ & $7.0 \pm 1.9$ & $6.1 \pm 1.2$ & $p<0.05$ \\
\hline MAPSE $(\mathrm{mm})$ & $12.9 \pm 2.7$ & $14.6 \pm 4.1$ & $p=n . s$. \\
\hline LV EDV/BSA $\left(\mathrm{ml} / \mathrm{m}^{2}\right)$ & $85.7 \pm 17.5$ & $73.6 \pm 14.2$ & $p<0.01$ \\
\hline LV ESV/BSA $\left(\mathrm{ml} / \mathrm{m}^{2}\right)$ & $31.8 \pm 15.2$ & $23.8 \pm 8.3$ & $p<0.05$ \\
\hline LV SV/BSA $\left(\mathrm{ml} / \mathrm{m}^{2}\right)$ & $53.9 \pm 9.6$ & $49.8 \pm 7.0$ & $p=n . s$. \\
\hline RV EF (\%) & $52.9 \pm 9.0$ & $60.2 \pm 7.4$ & $p<0.01$ \\
\hline RV EDV $(\mathrm{ml})$ & $178.0 \pm 29.5$ & $127.1 \pm 34.6$ & $p<0.0001$ \\
\hline RV ESV (ml) & $83.8 \pm 20.6$ & $50.8 \pm 17.3$ & $p<0.0001$ \\
\hline RV SV (ml) & $94.2 \pm 24.0$ & $76.3 \pm 21.2$ & $p<0.01$ \\
\hline $\begin{array}{l}\text { RV Cardiac output (I/ } \\
\text { min) }\end{array}$ & $6.8 \pm 2.1$ & $5.3 \pm 1.6$ & $p<0.01$ \\
\hline TAPSE $(\mathrm{mm})$ & $23.3 \pm 6.0$ & $20.1 \pm 4.7$ & $p<0.05$ \\
\hline RV EDV/BSA $\left(\mathrm{ml} / \mathrm{m}^{2}\right)$ & $52.5 \pm 11.5$ & $43.5 \pm 10.9$ & $p<0.01$ \\
\hline RV ESV/BSA $\left(\mathrm{ml} / \mathrm{m}^{2}\right)$ & $47.2 \pm 12.7$ & $28.9 \pm 8.8$ & $p<0.0001$ \\
\hline $\mathrm{RV} S \mathrm{~S} / \mathrm{BSA}\left(\mathrm{ml} / \mathrm{m}^{2}\right)$ & $52.5 \pm 11.5$ & $43.5 \pm 10.9$ & $p<0.001$ \\
\hline Wall mass (g) & $73.1 \pm 19.2$ & $63.3 \pm 20.9$ & $p=n . s$. \\
\hline $\begin{array}{c}\text { Wall mass/BSA (g/ } \\
\left.\mathrm{m}^{2}\right)\end{array}$ & $40.7 \pm 9.0$ & $35.9 \pm 9.5$ & $p=n . s$. \\
\hline
\end{tabular}

Table 2 Demographic and clinical characteristics

\begin{tabular}{|c|c|c|c|}
\hline & $\begin{array}{l}\text { SLE patients } \\
\qquad(\mathrm{n}=29)\end{array}$ & $\begin{array}{l}\text { Healthy controls } \\
\qquad(\mathrm{n}=30)\end{array}$ & \\
\hline Age (years) & $44.7 \pm 13.4$ & $44.7 \pm 11.1$ & $\begin{array}{c}p=n . \\
s\end{array}$ \\
\hline Sex (female) & 26/29 (89.7\%) & 27/30 (90.0\%) & $\begin{array}{c}p=n . \\
s\end{array}$ \\
\hline Weight (kg) & $71.6 \pm 15.2$ & $66.8 \pm 11.2$ & $\begin{array}{c}p=n . \\
s\end{array}$ \\
\hline $\mathrm{BMI}\left(\mathrm{kg} / \mathrm{m}^{2}\right)$ & $25.9 \pm 4.7$ & $23.9 \pm 4.0$ & $\begin{array}{c}p=n . \\
s\end{array}$ \\
\hline $\mathrm{BSA}\left(\mathrm{m}^{2}\right)$ & $1.79 \pm 0.19$ & $1.74 \pm 0.15$ & $\begin{array}{c}p=n . \\
s\end{array}$ \\
\hline Heart rate (bpm) & $70.9 \pm 12.6$ & $66.5 \pm 9.5$ & $\begin{array}{c}p=n . \\
s\end{array}$ \\
\hline $\begin{array}{l}\text { Blood pressure systolic } \\
\qquad(\mathrm{mmHg})\end{array}$ & $122.7 \pm 10.7$ & $123.8 \pm 15.8$ & $\begin{array}{c}p=n . \\
s\end{array}$ \\
\hline $\begin{array}{l}\text { Blood pressure diastolic } \\
\qquad(\mathrm{mmHg})\end{array}$ & $74.2 \pm 11.5$ & $75.6 \pm 6.9$ & $\begin{array}{c}p=n . \\
s\end{array}$ \\
\hline
\end{tabular}

\section{Author details}

'Department of Cardiology, University of Heidelberg, Heidelberg, Germany. ${ }^{2}$ School of Medicine of Ribeirao Preto University of Sao Paulo, Sao Paulo, Brazil.

Published: 30 January 2013
doi:10.1186/1532-429X-15-S1-P94

Cite this article as: Andre et al:: Prevalence of cardiac morphological and functional alterations in systemic lupus erythematosus patients with a low disease activity. Journal of Cardiovascular Magnetic Resonance 2013 15(Suppl 1):P94.

\section{Submit your next manuscript to BioMed Central} and take full advantage of:

- Convenient online submission

- Thorough peer review

- No space constraints or color figure charges

- Immediate publication on acceptance

- Inclusion in PubMed, CAS, Scopus and Google Scholar

- Research which is freely available for redistribution

Submit your manuscript at www.biomedcentral.com/submit
C Biomed Central 